Tohoku J. Exp. Med., 2007, 212, 81-90

\title{
Development of Glomerular Endothelial Cells, Podocytes and Mesangial Cells in the Human Fetus and Infant
}

\author{
Kei Takano, ${ }^{1}$ Yukihiko Kawasaki, ${ }^{1}$ Tomoko Imaizumi, ${ }^{1}$ Hiromi Matsuura, ${ }^{1}$ \\ Ruriko Nozawa, ${ }^{1}$ Mieko Tannis, ${ }^{1}$ Kazuhide Suyama, ${ }^{1}$ Masato Isome, ${ }^{1}$ \\ Hitoshi Suzuki ${ }^{1}$ and Mitsuaki Hosoya ${ }^{1}$ \\ ${ }^{1}$ Department of Pediatrics, Fukushima Medical University School of Medicine, Fukushima, \\ Japan
}

Takano, K., Kawasaki, Y., Imaizumi, T., Matsuura, H., Nozawa, R., Tanni, M., Suyama, K., Isome, M., Suzuki, H. and Hosoya, M. Development of Glomerular Endothelial Cells, Podocytes and Mesangial Cells in the Human Fetus and Infant. Tohoku J. Exp. Med., 2007, 212 (1), 81-90 — The process of glomerular development consists of four developmental stages: vesicle (V) stage, S-shaped body (S) stage, capillary loop (C) stage and maturation (M) stage. However, the development of glomerular endothelial, mesangial and epithelial cells in fetal and infant kidneys remains unclear. In order to determine the characteristics of human glomerular development, we investigated the process of glomerular development by staining fetal and infant kidneys for CD31, CD34 and FB21, markers for endothelial cells, $\alpha$-smooth muscle actin ( $\alpha$-SMA), a marker for mesangial cells, and nephrin, a marker for podocytes. These series of studies were carried out on kidneys obtained at autopsy from 27 fetuses and 5 infants. The fetuses were divided into the following 5 groups according to gestational age; $13-19,20-24,25-29,30-34$ and 35-39 weeks. In each group, glomerular development was classified according to the developmental stage and the staining patterns for CD31, CD34, FB21, $\alpha$-SMA and nephrin. The proportion of $\mathrm{V}$-stage development in 100 glomeruli examined was highest at 13-19 weeks. After 20 weeks, the V-stage proportion decreased gradually, and the proportion of S stage became highest at 20-24 weeks. The C-stage proportion was highest at 25-29 weeks, while the M-stage proportion was highest in infants aged 1-6 months. The staining patterns for CD31, CD34 and FB21 were similar in endothelial cells after 25 weeks of gestation. Staining of $\alpha$-SMA and nephrin was first observed in the S stage. In conclusion, maturation of endothelial cells starts at 25 weeks and is completed by 35 weeks of gestation. Epithelial cells and mesangial cells first appear during the $\mathrm{S}$ stage. —— glomerular development; CD31; CD34; FB21

(C) 2007 Tohoku University Medical Press

The glomerulus, the most important filtering apparatus in the body, is a unique and highly specialized structure. Three types of cells in the glo- merular tufts, the glomerular capillary endothelial cells, mesangial cells and glomerular epithelial cells, can be clearly identified, and their functions

Received December 22, 2006; revision accepted for publication April 2, 2007.

Correspondence: Yukihiko Kawasaki, M.D., Department of Pediatrics, Fukushima Medical University School of Medicine, 1 Hikariga-oka, Fukushima City, Fukushima 960-1295, Japan.

e-mail: kei-1972@lapis.plala.or.jp 
are now well understood. However, the features of glomerulogenesis remain controversial. Fetal glomerular features change drastically during development; thus it is very difficult to distinguish each cell type in the developing glomeruli.

The process of glomerular development consists of four developmental stages: vesicle (V) stage, S-shaped body (S) stage, capillary loop (C) stage and maturing $(\mathrm{M})$ stage. It has been reported that the glomeruli increase in maturity while progressing from $\mathrm{V}$ to $\mathrm{M}$ stage. However, the proportions of these stages during the process of glomerular development in fetal and infant kidneys are still unclear (Reeves et al. 1978; Aperia et al. 1980; Hayslett 1983; Dinkel et al. 1985; Saxen and Sariola 1987; Sherer et al. 1990; Elger et al. 1991).

In this study, to reveal the process of human glomerular development, and development of three glomerular cells in the glomerular tufts, the endothelial cells especially, we evaluated the developmental processes of glomeruli and three gulomerular cells using immunohistochemistry in fetal and infant kidneys.

\section{Patients and Methods}

\section{Patients}

This study was performed on the kidneys of 27 fetuses and 5 infants with normal kidneys obtained at autopsy at Fukushima Medical University School of Medicine between January 1993 and June 2005.

Fetal age was estimated from gestational age and ranged from 13 to 39 weeks (13 to 19 weeks, Group A $[n=3] ; 20$ to 24 weeks, Group B [ $n=7] ; 25$ to 29 weeks, Group C [ $n=7]$; 30 to 34 weeks, Group D [ $n=6]$, 35 to 39 weeks, Group E $[n=4])$. Infant age ranged from 1 to 6 months (Group F $[n=5]$ ). The body weights of fetuses and infants were within the range of the mean \pm 1.5 S.D. of the body weights of normally developing fetuses and infants. The causes of death for fetuses and infants were induced and spontaneous abortion, fetal death and sudden infant death syndrome. None of the renal tissue specimens from these fetuses and infants exhibited macroscopic or microscopic evidence of pathology. We evaluated the process of glomerular development using four developmental stages (V, S, C and M stages) and three cell types of glomeruli: endothelial cells by staining for CD31, CD34 and FB21, mesangial cells by staining for $\alpha$-smooth muscle actin ( $\alpha$-SMA), and podocytes cells by staining for nephrin in fetuses and infants with normal kidneys.

\section{Pathological characteristics}

The specimens were assessed by light microscopy (LM) and immunohistochemically. Material for histological study was fixed in $20 \%$ neutral formalin, embedded in paraffin, cut into $2-3 \mu \mathrm{m}$ sections, and stained with hematoxylin and eosin or periodic acid-Schiff regent. Immunohistochemical detection of CD31, CD34, FB21, $\alpha$-SMA and nephrin was performed on paraffin sections by the enzyme-labeled indirect method. The primary antibodies used were mouse anti-human CD31 monoclonal antibody (Chemicon International, Temecula, CA, USA), mouse anti-human CD34 monoclonal antibody (NU-4A1; $1: 50$; HISTOFINE, Japan), mouse monoclonal antibody FB21 against $\alpha 2,6$-sialylconjugates (provided by Dr. Y Nozawa), $\alpha$-SMA monoclonal antibody (1:50; Dakopatts, Glostrup, Denmark), and guinea pig polyclonal antibody nephrin (PRPGEN Biotechnik $\mathrm{GmbH})$. Peroxidase-conjugated rabbit antimouse $\mathrm{IgG}$ (1 : 100; Dakopatts) for CD31, CD34, FB21, and $\alpha$-SMA and goat antiporcine IgG (1:100; Dakopatts) for nephrin were used as the secondary antibody. Endogenous peroxidase activity was blocked with $0.3 \%$ hydrogen peroxide in methanol for $30 \mathrm{~min}$ at room temperature. The sections were washed in phosphate-buffered saline (PBS) and then incubated overnight at $4^{\circ} \mathrm{C}$ with primary antibody. After washing again with $\mathrm{PBS}$, the sections were incubated overnight at $4{ }^{\circ} \mathrm{C}$ with secondary antibody. After a final wash with PBS, the sections were reacted in substitute medium containing 3,3'-diaminobenzidine tetrachloride $(\mathrm{DAB})$ before nuclear staining with hematoxylin. The primary antibody was replaced by a comparable immunoglobulin to provide a negative control.

\section{Light microscopy}

In order to determine the quantitative proportion of the four developmental stages of glomerular formation, we individually counted these stages in 100 glomeruli from each specimen. The counted glomeruli were evenly distributed from the deep zone of kidney to the superficial cortex.

\section{Immunohistochemistry}

The degree of CD31, CD34 and FB21 staining of each glomerulus was graded according to the following scale (Figs 1, 2 and 3): 0 = negative; 1 = weak; 2 = mod- 


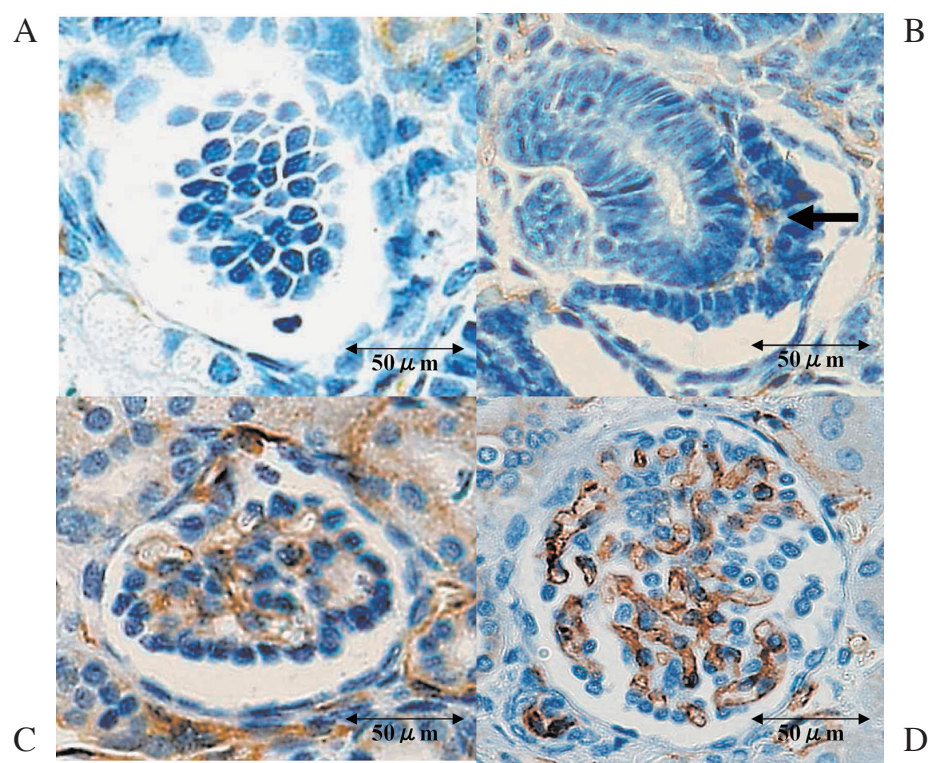

Fig. 1. CD31 staining of glomerular endothelial cells on light microscopy.

A: Score 0: There was no CD31 staining in 17-week fetal kidneys $(\times 400)$, and a mesenchymal cell cluster had been formed in the nephrogenic zone. B: Score 1: In fetal kidney after 20 weeks, CD31 was weakly reactive with glomerular endothelial cells and the differentiated lower limb of the S-shaped body had formed an immature glomerulus $(\times 400)$. C: Score 2: In 30-week fetal kidney, they had migrated to the periphery of the glomerular tuft $(\times 400)$. CD31 exhibited a distinct pattern of localization in the developing human kidney. D: Score 3: CD31 was reactive with glomerular endothelial cells in the kidneys of fetuses older than 35 weeks $(\times 400)$.

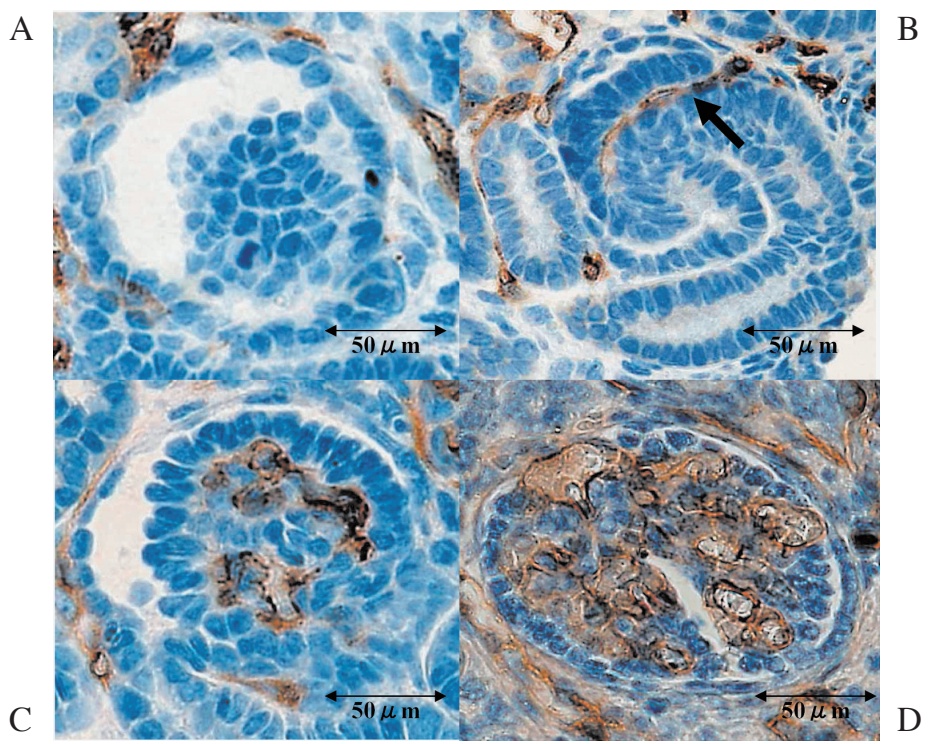

Fig. 2. CD34 staining of glomerular endothelial cells on light microscopy.

A: Score 0: We found no reactivity with CD34 in the fetal kidney before 20 weeks $(\times 400)$. B: Score 1: CD34 was moderately reactive with glomerular endothelial cells in 27-week fetal kidney $(\times 400)$. C: Score 2: CD34 was reactive with glomerular endothelial cells in the fetal kidney after 30 weeks $(\times 400)$. D: Score 3: CD34-positive glomerular endothelial cells subsequently aggregated at the root of the developing glomerulus, and had migrated to the periphery of the glomerular tuft in the 35-week fetal kidney ( $\times 400)$. 


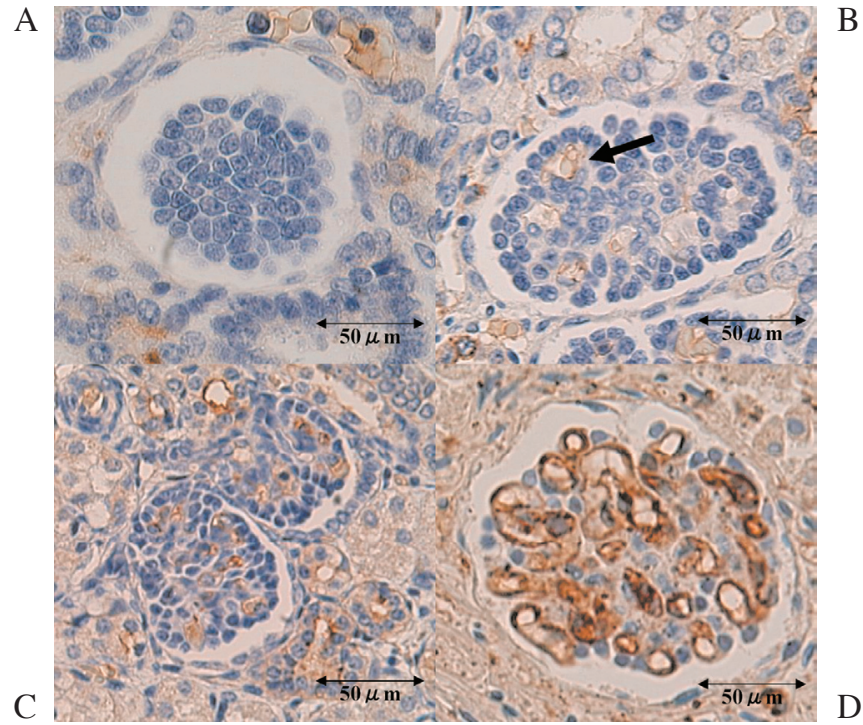

Fig. 3. FB21 staining of glomerular endothelial cells on light microscopy.

A: Score 0: There was no FB21 staining in 20-week fetal kidneys, and a mesenchmal cell cluster had been formed in the nephrogenic zone $(\times 400)$. B: Score 1: There was weak FB21 staining in an immature glomerulus formed in 24-week fetal kidneys $(\times 400)$. C: Score 2: FB21 was moderately reactive with glomerular endothelial cells in 27-week fetal kidneys $(\times 400)$. D: Score 3: FB21strongly positive glomerular endothelial cells aggregated at the root of developing glomerulus and then migrated to the periphery of the glomerular tuft, in 37-week fetal kidneys $(\times 400)$.

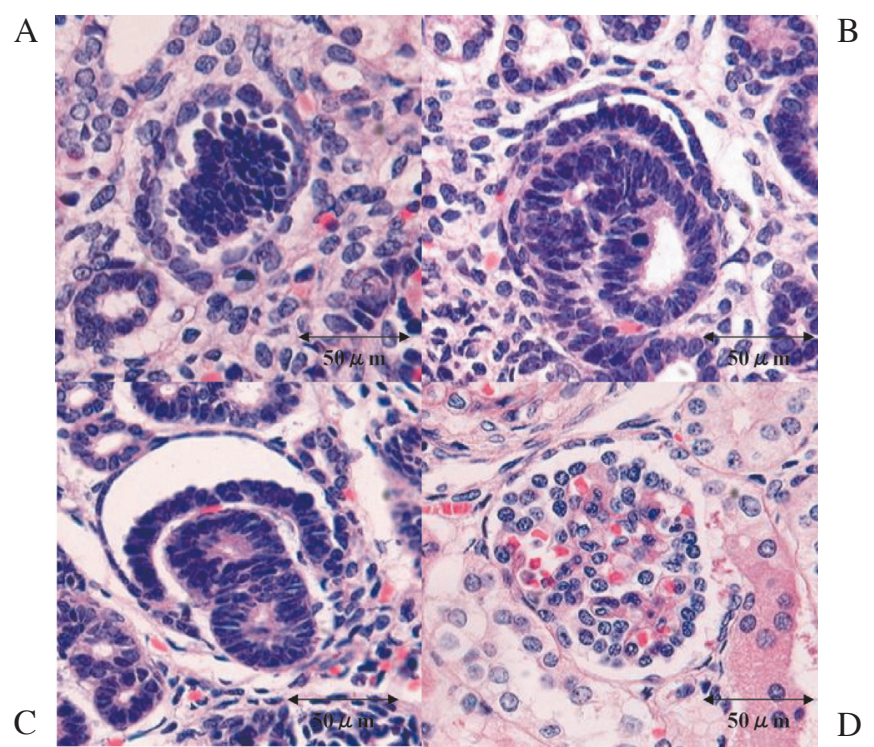

Fig. 4. Findings for V-stage, S-stage, C-stage and M-stage.

A: V-stage glomeruli are evident in this 17 -week fetal kidney $(\times 400)$. In the initial stage of glomerular development, a cluster of primitive mesenchymal cells is formed in the nephrogenic zone. B: S-stage glomeruli are evident in this 24 -week fetal kidney $(\times 400)$. The vesicle then gradually elongates, twists and is transformed from a comma-shaped body into an S-shaped body with a narrow lumen. C: C-stage glomeruli are evident in this 27 -week fetal kidney $(\times 400)$. The differentiated lower limb of the S-shaped body forms an immature glomerulus. D: M-stage glomeruli are evident in this 37 -week fetal kidney $(\times 400)$. The immature glomerulus enlarges, and the numerous capillary loops form complicated glomerular tufts and develop into the mature glomerulus. 
erate; $3=$ strong. Staining scores were totaled and divided by the number of glomeruli to obtain the mean score, which was recorded as the value for the patient. $\alpha$-SMA and nephrin staining was evaluated as a phenotypic transformation of mesangial cells and epithelial cells on four stages. The histological tissues were reviewed by two independent investigators who were unaware of the clinical data of patients in the study.

\section{Statistics}

Values are the mean \pm S.E.M. Statistical analysis was performed on a Windows XP computer with a software package for statistical analysis (Stat View, Abacus Concepts, Berkeley, CA, USA). Differences in laboratory data between groups were assessed by ANOVA. Correlations were assessed using Fisher's r-test. $P$ values less than 0.05 were considered significant.

\section{RESULTS}

Changes in renal developmental stage in fetal and infant kidneys

The typical pathological findings for V-stage, S-stage, C-stage and M-stage are shown in Fig. 4. The changes in proportions of the four glomerular developmental stages in fetal and infant kidneys are shown in the Fig. 5. V-stage proportion was significantly higher in Group A (13 to 19 weeks) than in Group B (20 to 24 weeks) $(p<0.05)$ and the other groups. In fetal kidney after 30 weeks, $\mathrm{V}$-stage proportion gradually decreased with time, but V-stage still remained in Group F ( 1 to 6 months). S-stage proportion was significantly higher in Group B (20 to 24 weeks) than in Group $\mathrm{E}$ (35 to 39 weeks) $(p<0.01)$ and F (1 to 6 months) $(p<0.01)$. C-stage proportion was significantly higher in Group C (25 to 29 weeks) than in Group F ( 1 to 6 months) $(p<0.05)$. In fetal kidney after 30 weeks, S-stage and C-stage proportions gradually decreased. In infant kidney after 40 weeks, S-stage and C-stage were rarely detected. M-stage proportion was significantly higher in Group F (1 to 6 months) than that in each group of fetal kidneys $(p<0.01)$.

Evaluation of developing endothelial cells in fetal and infant kidney: CD31, CD34 and FB21 staining

The glomerular CD31, CD34 and FB21 staining scores in each group are shown in Fig. 6. The mean glomerular CD31 staining scores in Group A through F were $0.0 \pm 0.0$ (13 to 19

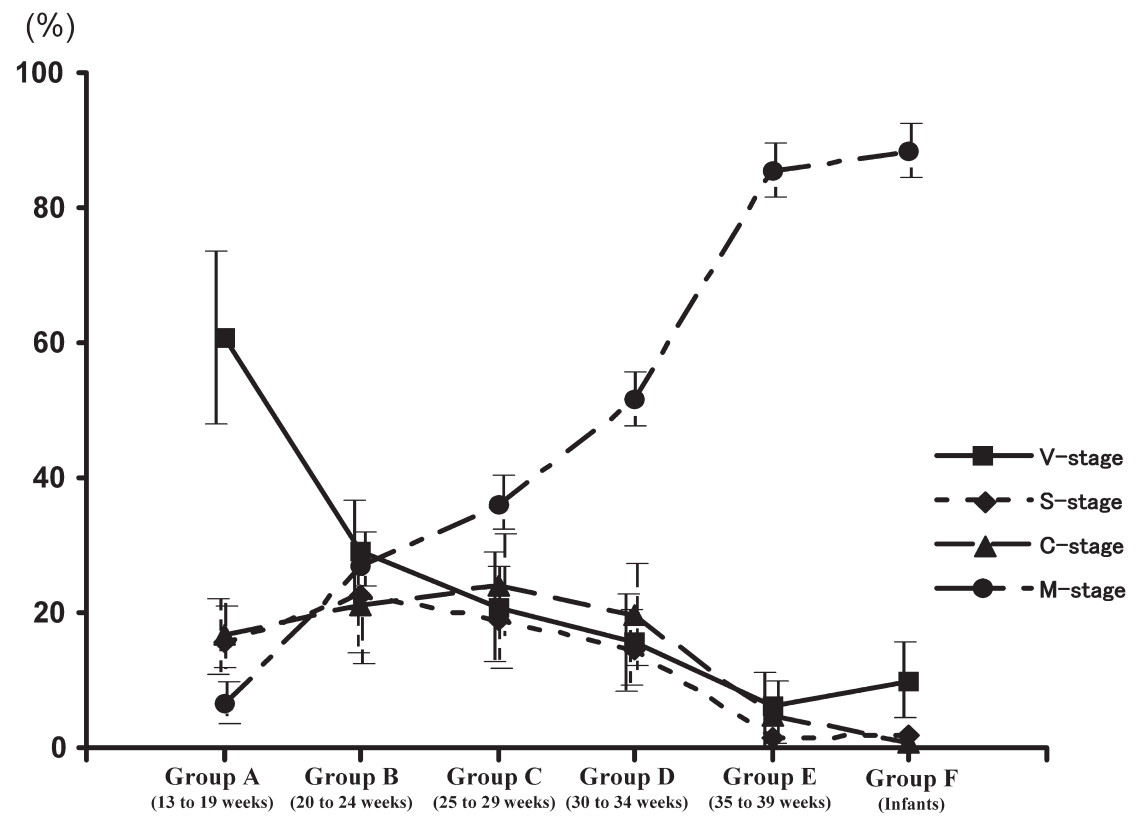

Fig. 5. Changes in proportions of renal developmental stages in each age group.

, proportion of V-stage;

, proportion of S-stage;

$\mathbf{A}$, proportion of $\mathrm{C}$-stage;

, proportion of M-stage. 


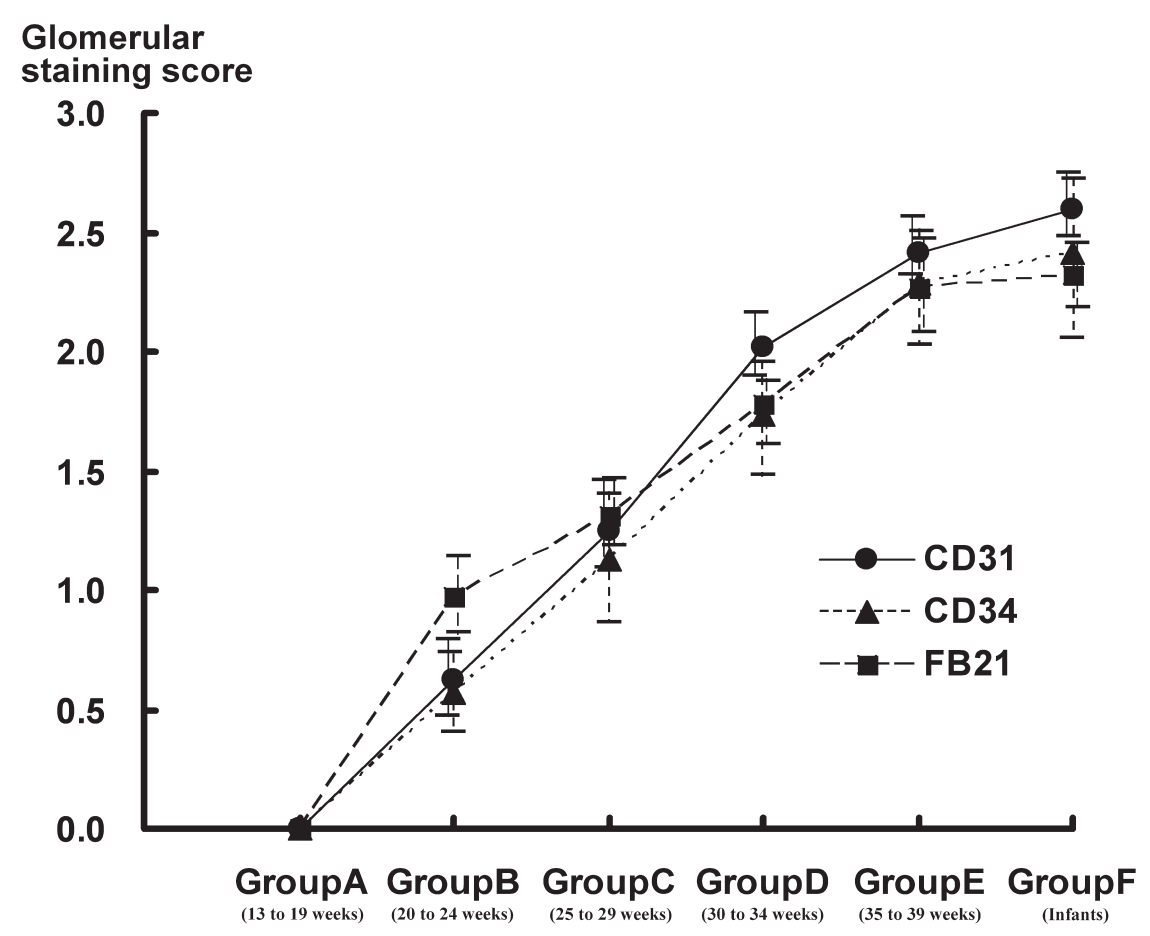

Fig. 6. Comparison of glomerular CD31-, CD34- and FB21-staining scores in each age group. , staining score of CD31; $\boldsymbol{\Delta}$, staining score of CD34; $\boldsymbol{\square}$, staining score of FB21.

weeks), $0.62 \pm 0.21$ (19 to 24 weeks), $1.24 \pm 0.19$ ( 25 to 29 weeks), $2.02 \pm 0.12$ (30 to 34 weeks), $2.41 \pm 0.18$ ( 35 to 39 weeks) and $2.59 \pm 0.21$ ( 1 to 6 months), respectively. The mean glomerular CD34 staining scores were $0.0 \pm 0.0$ (13 to 19 weeks), $0.56 \pm 0.27$ (20 to 24 weeks), $1.12 \pm 0.48$ ( 25 to 29 weeks), $1.73 \pm 0.44$ (30 to 34 weeks), $2.28 \pm 0.43$ (35 to 39 weeks) and $2.41 \pm 0.55$ ( 1 to 6 months), respectively. The mean glomerular FB21 staining scores in Group A through F were $0.0 \pm 0.0$ (13 to 19 weeks), $0.97 \pm 0.23$ (20 to 24 weeks), $1.31 \pm 0.14$ ( 25 to 29 weeks), $1.77 \pm 0.21$ (30 to 34 weeks), $2.26 \pm 0.31$ (35 to 39 weeks) and $2.32 \pm 0.11$ ( 1 to 6 months), respectively. There was no detectable CD31, CD34 and FB21 staining in the 13-week fetal kidney, in which mesenchymal cell clusters had been formed in the nephrogenic zone. Antibodies to CD31, CD34 and FB21 were weakly reactive with glomerular endothelial cells in fetal kidney after 20 weeks. At this age the differentiated lower limb of the S-shaped body formed an immature glomerulus. CD31-, CD34- and FB21-positive glomerular en- dothelial cells aggregated at the roots of developing glomeruli in the 25 -week fetal kidney. These cells had migrated to the periphery of the glomerular tuft in 30-week fetal kidney. CD31, CD34 and FB21 exhibited a distinct pattern of localization in the developing human kidney. CD31, CD34 and FB21 were positive in glomerular endothelial cells of the kidneys of fetuses older than 35 weeks. The glomerular staining score of CD31 was comparable with the score for CD34. The FB21 staining score was also comparable to that of CD31 and CD34 for endothelial cells in the kidneys of fetuses at similar weeks of gestation.

Evaluation of mesangial cell development in fetal and infant kidney: $\alpha$-SMA staining

In the V-stage, the cells of the vesicle were negative for $\alpha$-SMA staining. $\alpha$-SMA-positive mesenchymal cells were found in the lower cleft of the S-shaped body (Fig. 7). In the late C-stage, $\alpha$-SMA-positive mesenchymal cells appeared to migrate toward the periphery of the immature 


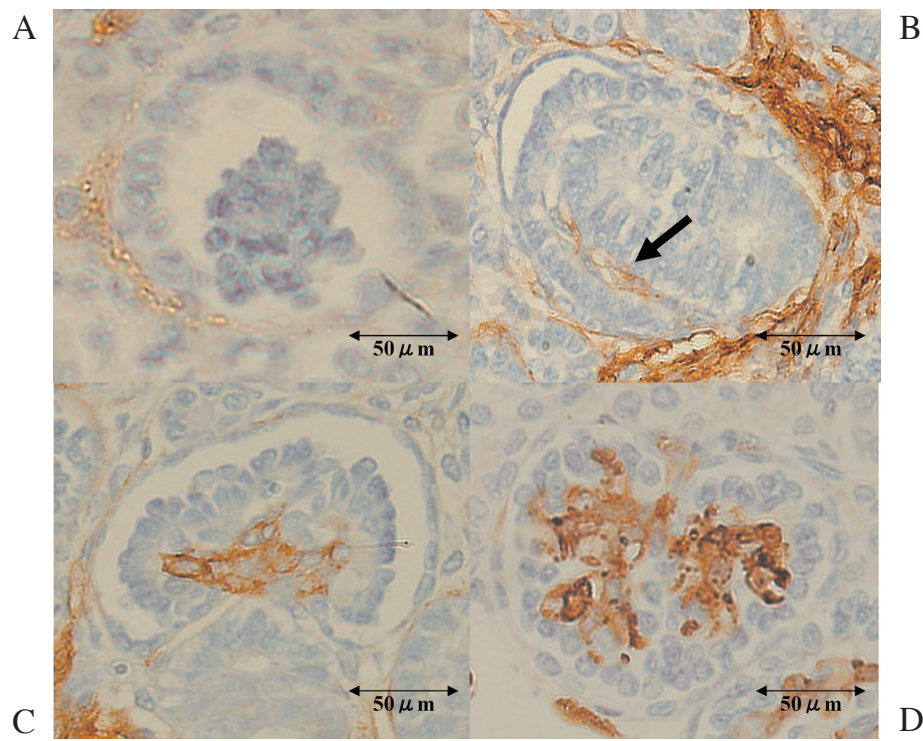

Fig. 7. Staining for $\alpha$-SMA.

A: At the V-stage in 17-week fetal kidneys $(\times 400)$, the cells of the vesicle were negative for $\alpha$-SMA staining. B: At the S-stage in 24-week fetal kidneys ( $\times 400), \alpha$-SMA-positive mesenchymal cells are located in the lower cleft of the S-shaped body. C: At the C-stage in 27-week fetal kidneys ( $\times 400), \alpha$-SMA-positive mesenchymal cells appeared to migrate toward the periphery of the immature glomerulus. D: At the M-stage in 37-week fetal kidneys ( $\times 400), \alpha$-SMA-positive mesenchymal cells aggregate at the root of developing glomerulus and then migrate to the periphery of the glomerular tuft.

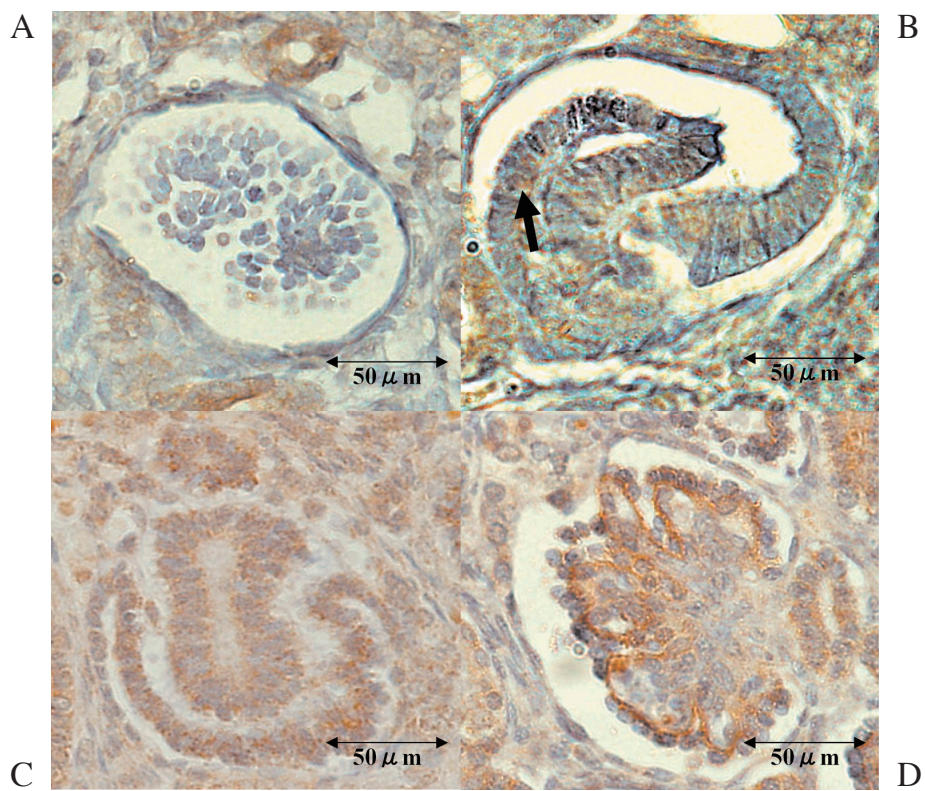

Fig. 8. Staining for nephrin.

A: No staining for nephrin was found in the V-stage in 17-week fetal kidneys $(\times 400)$. B: Weak nephrin signal is first observed in S-shaped bodies in 24-week fetal kidneys $(\times 400)$, specifically in columnar epithelial cells adjacent to the vascular cleft. C: In the C-stage glomeruli in 27 -week fetal kidneys ( $\times 400)$, nephrin is found between developing podocytes as dots or discrete lines. D: In the M-stage glomeruli in 37-week fetal kidneys ( $\times 400)$, nephrin is distributed exclusively along the glomerular basement membrane. 
glomerulus. In the M-stage, $\alpha$-SMA mesenchymal cells were scattered and exclusively located in the mesangium.

Evaluation of epithelial cell development in fetal and infant kidney: Nephrin staining

No staining for nephrin was found in the $\mathrm{V}$-stage. Weak nephrin signal was first observed in S-shaped bodies, specifically in columnar epithelial cells adjacent to the vascular cleft (Fig. 8). In the C-stage glomeruli, nephrin was found between developing podocytes as dots or discrete lines. In the M-stage glomeruli, nephrin was distributed exclusively along the glomerular basement membrane.

\section{Discussion}

We found that V-stage proportion was higher in Group A (13 to 19 weeks) than in the other groups. In fetal kidney after 20 weeks, V-stage proportion gradually decreased. S-stage and C-stage proportions were higher in Groups B (20 to 24 weeks) and C (25 to 29 weeks) than in the other groups. In fetal kidney after 30 weeks, $\mathrm{S}$-stage and C-stage proportions gradually decreased. In infant kidney after 40 weeks, S-stage and C-stage were rarely present and most glomeruli were in M-stage. M-stage proportion was higher in Group F (1 to 6 months) than in all other groups.

It has been reported that the glomerulus increases in maturity while progressing from $\mathrm{V}$-stage to M-stage. There have been a few previous reports on the proportions of the four stages in the process of glomerular development in fetal and infant kidneys (Reeves et al. 1978; Aperia et al. 1980; Hayslett et al. 1983; Dinkel et al. 1985; Saxen et al. 1987; Sherer et al. 1990; Elger et al. 1991).

In our study, the proportion of V-stage was higher in Group A (13 to 19 weeks) than in the other groups. In fetal kidney after 20 weeks, $\mathrm{V}$-stage proportion gradually decreased, but V-stage remained in Group F (1 to 6 months). Glomerulogenesis is associated with glomerular obsolescence. There are no immature gulomeruli in renal tissue of adolescent and adult. Therefore it is possible that the $\mathrm{V}$-stage glomeruli that remained in infants (1 to 6 months) may disappear with time as a result of the induction of glomerular apoptosis. S-stage and C-stage proportions were higher in Group B (20 to 24 weeks) and C (25 to 29 weeks) than in the other groups. In fetal kidney after 30 weeks, S-stage and C-stage proportions gradually decreased. In infant kidney after 40 weeks, S-stage and C-stage were rarely present and most glomeruli were in M-stage. $\mathrm{M}$-stage proportion was higher in Group $\mathrm{F}$ than in all other groups. These findings suggest that the glomerulus may gradually mature to $\mathrm{M}$-stage from V-stage over weeks 20-34 of gestation, and that immature glomeruli such as those of V-stage remain in the infant kidney.

Three types of cells in the glomerular tufts, the glomerular capillary endothelial cells, mesangial cells, and glomerular epithelial cells, can be clearly identified, and their functions are now well understood. However, the features of glomerulogenesis remain controversial.

Glomerular endothelial cells are active participants in the processes controlling coagulation, inflammation, and immune processes, and synthesize and release endothelin-1 and endotheliumderived relaxing factor. Thus, it has been suggested that glomerular endothelial cells play an important role in maintaining renal homeostasis (Chester 1992; Charles et al. 1998; Barry 2002). However, the glomerular endothelial cell development was still obscure and there have been few reports on the features of glomerular endothelial cells in developing glomeruli. On the other hand, as indices of glomerular endothelial cell injury, several markers, such as CD31, CD34, and FB21 have been used. CD31 is a ligand for PECAM-1 (platelet endothelial cell adhesion molecule 1) and is expressed by vascular endothelial cells. It plays a major role in the adhesion cascade between endothelial cells and inflammatory cells during the evolution of inflammation, facilitates leukocyte emigration between endothelial cells and is involved in angiogenesis. CD34 is a ligand for L-selectin and is expressed by vascular endothelial cells (Ricono et al. 2003; Pusztaszeri et al. 2006). FB21 is a monoclonal antibody that binds 
a sialic-acid-dependent surface antigen and reacts with human glomerular endothelial cells (Nozawa et al. 1993, 1994; Kawasaki et al. 2004). We previously reported that FB21 reacted with the glomerular endothelial cells of normal kidneys and was detected on the surface of glomerular endothelial cells, and noted that FB21 staining of glomerular endothelial cell was significantly weaker in patients with hemolytic uremic syndrome (HUS) and Henoch-Schoenlein purpura nephritis (HSPN) than in children with normal kidneys, and indicated that FB21 can be used as a marker for glomerular endothelial cell injury and dysfunction in HUS and HSPN patients (Kawasaki et al. 2004).

In the present study, CD31 was reactive with glomerular endothelial cells in the fetal kidney after 25 weeks. CD31-positive glomerular endothelial cells subsequently aggregated at the roots of the developing glomeruli, and had migrated to the periphery of the glomerular tuft in the 35-week fetal kidney. These findings suggest that maturation of the endothelial cells starts at about 25 weeks in the fetus and that these cells have matured in the fetus by 35 weeks.

CD34 was not reactive in the fetal kidney before 20 weeks, but CD34 was reactive with glomerular endothelial cells in the fetal kidney after 30 weeks. CD34-positive glomerular endothelial cells subsequently aggregated at the root of the developing glomerulus, and had migrated to the periphery of the glomerular tuft in the 35-week fetal kidney. These findings suggest that maturation of the endothelial cells starts at week 25 in the fetus and that these cells are mature by $35-39$ weeks in the fetus.

In our study, glomerular staining score of FB21 was similar to that of CD31 and CD34 for endothelial cells in the kidneys of fetuses in each week of gestation. Therefore, FB21 that reacts with a sialic-acid-dependent carbohydrate epitope can be a maker for development of glomerular endothelial cell. Westley et al. (1980) described that the appearance of sialoglycoproteins on the epithelial cell surface coincides with the development of foot processes and filtration slits. The appearance of a sialic-acid on the endothelial cell surface may indicate functional development of the endothelial cell.

On the other hand, together with the mesangial matrix, the mesangial cells constitute the glomerular mesangium. Mesangial cells are quite irregular in shape, with many processes extending from the cell body toward the glomerular basement membrane. In these processes, dense assemblies of microfilaments are found, which have been shown to contain actin, myosin and actinin. The mesangial cells are important targets in many glomerular immune and nonimmune diseases and characteristically respond by injury, repair, and proliferation. While no definite markers for mesangial cells have yet been found, a few studies have reported that human and rat fetal mesangial cells are positive for $\alpha$-SMA, compared with the negative immunostaining in adult mesangial cells(Kawasaki et al. 2003). The vesicle structure (V) contains positive mesenchymal cells. $\alpha$-SMA-positive mesenchymal cells are located in the lower cleft of the S-shaped body. $\alpha$-SMA-positive mesenchymal cells appeared to migrate toward the periphery of the immature glomerulus. $\alpha$-SMA-positive mesenchymal cells aggregate at the root of developing glomerulus and then migrate to the periphery of the glomerular tuft. In our study, $\alpha$-SMA-stained mesangial cells were found in the lower clefts of the $\mathrm{S}$-shaped bodies first of all.

The visceral epithelium of Bowman's capsule consists of highly differentiated cells, the podocytes. In the developing glomerulus, podocytes are of a simple polygonal shape. Podocytes are specialized glomerular epithelial cells of the kidney. Their interdigitating foot processes surround the glomerular capillary wall facing the urinary space. The specialized intercellular junction between podocyte foot processes, called the slit diaphragm, serves as an essential component of the glomerular filter. Nephrin is a cell adhesion protein located at the slit diaphragm area of glomerular podocytes (Vesa et al. 2000). Immunoperoxidase staining for nephrin in normal glomerulogenesis using antibodies directed against the intracellular part of nephrin. Weak nephrin signal is first observed in late $\mathrm{S}$-shaped 
bodies, specifically in columnar epithelial cells adjacent to the vascular cleft. In capillary stage glomeruli, nephrin is found along the basal margin and between developing podocytes as dots or discrete lines. Inset shows two discrete lines of nephrin-specific peroxidase between developing podocytes. In maturing stage glomeruli, nephrin is distributed exclusively along the glomerular basement membrane. In our study, nephrin stained epithelial cells were first observed weakly in the S-shaped bodies.

In conclusion, the glomerulus matures to M-stage from V-stage between 25-34 weeks gestation. Maturation of endothelial cells starts at 25 weeks and is completed at 35 weeks. Epithelial cells and mesangial cells appear first at the S-stage of development.

\section{References}

Aperia, A., Larsson, L. \& Wilton, P. (1980) The effect of normal development on compensatory renal growth. Kidney Int., 18, 29-35.

Barry, M.B. (2002) Brenner \& Rector's THE KIDNEY, 15th ed., Saunders, Philadelphia, pp. 72-98.

Charles, J., Jean, L., Melvin, M. \& Fred, G. (1998) Heptinstall's Pathology of the Kidney, 5th ed., Lippincott-Raven, Philadelphia, pp. 67-84.

Chester, M.E. (1992) Pediatric Kidney Disease, 2nd ed., Little Brown, Boston, pp. 19-48.

Dinkel, E., Ertel, M. \& Dittrich, M. (1985) Kidney size in childhood. Sonographical growth charts for kidney length and volume. Pediatr. Radiol., 15, 38-43.

Elger, M., Sakai, T., Winkler, D. \& Kriz, W. (1991) Structure of the outflow segment of the efferent arteriole in rat superficial glomeruli. Contrib. Nephrol., 95, 22-33.

Hayslett, J.P. (1983) Effect of age on compensatory renal growth. Kidney Int., 23, 599-602.

Kawasaki, Y., Suzuki, J., Sakai, N., Tannji, M. \& Suzuki, H. (2003) Predicting the prognosis of renal dysfunction by renal expression of $\alpha$-smooth muscle actin in children with MPGN type 1. Am. J. Kidney Dis., 42, 1131-1138.

Kawasaki, Y., Suzuki, J., Nozawa, R., Sakai, N., Tannji, M., Isome, M., Suzuki, H. \& Nozawa, Y. (2004) FB21, a monoclonal antibody that reacts with a sialic-acid-dependent carbohydrate epitope, is a marker for glomerular endothelial cell injury. Am. J. Kidney Dis., 44, 239-249.

Nozawa, Y., Wachi, E., Tominaga, K., Abe, M. \& Wakasa, H. (1993) A novel monoclonal antibody (FUN-1) identified an activation antigen in cells of the B-cell lineage and Reed-Sternberg cells. J. Pathol., 169, 309-315.

Nozawa, Y., Abe, M., Ohno, H., Fukuhara, S. \& Wakasa, H. (1994) Production of two monoclonal antibodies (FB1 and FB21) useful for the identification of human B lymphocytes in formalin-fixed, paraffin-embedded tissues. $J$. Pathol., 173, 347-354.

Pusztaszeri, M., Chaubert, P. \& Seelentag, W. (2006) Immumohistochemical expression of endothelial markers CD31, CD34, von willebrand factor, and Fli-1 in normal human tissues. J. Histochem. Cytochem., 54, 385-395.

Reeves, W., Caulfield, J.P. \& Farquhar, M.G. (1978) Differentiation of epithelial foot processes and filtration slits. Lab. Invest., 39, 90-100.

Ricono, J.M., Xu, Y.C. \& Arar, M. (2003) Morphological insights into the origin of glomerular endothelial and mesangial cells and their precursors. J. Histochem. Cytochem., 51, 141-150.

Saxen, L. \& Sariola, H. (1987) Early organogenesis of the kidney. Pediatr. Nephrol., 3, 385-392.

Sherer, D.M., Thompson, H.O., Armstrong, B. \& Woods, J.R. (1990) Prenatal sonographic diagnosis of unilateral fetal renal agenesis. J. Clin. Ultrasound., 18, 648-652.

Vesa, R., Jaakko, P., Paivi, T., Paula, R., Michael, H., Marjo, K., Christer, H., Riitta, S., Markku, H., Jorma, W., Karl, T. \& Hannu, J. (2000) Role of nephrin in cell junction formation in human nephrogenesis. Am. J. Pathol., 157, 1905-1916.

Westly, H.R., Yashpal, S.K. \& Marilyn, G.F. (1980) Assembly of the glomerular filtration surface. J. Cell Biol., 85, 735-753. 